\title{
Dilution effect on microstructures with respect to magnetic and dielectric properties in charge-ordered ferroelectric $\mathrm{LuFe}_{2} \mathrm{O}_{4}$
}

\author{
Y.Matsuo, Y.Horibe ${ }^{1}$, K.Yoshii ${ }^{2}$, N.Ikeda ${ }^{3}$, S.Mori \\ Osaka Prefecture University, Sakai, Osaka 599-853, Japan \\ ${ }^{1}$ Rutgers University, Piscataway, NJ, 08854, U.S.A \\ ${ }^{2}$ Japan Atomic Agency, Hyogo 679-5148, Japan \\ ${ }^{3}$ Okayama University, Okayama 700-8530, Japan
}

\begin{abstract}
We investigated magnetic/dielectric properties and related microstructures in $\mathrm{LuFeCuO}_{4}$, which was obtained by substituting $\mathrm{Cu}^{2+}$ for $\mathrm{Fe}^{2+}$ in $\mathrm{LuFe}_{2} \mathrm{O}_{4}$. It was found that $\mathrm{LuFeCuO}_{4}$ shows a characteristic low-frequency dielectric dispersion around $300 \mathrm{~K}$. We analyzed it using the Debye model and found that $\mathrm{LuFeCuO}_{4}$ is a dielectric compound with an orientational polarization. Electron diffraction experiments revealed that there exists characteristic zigzag-shaped diffuse scattering in $\mathrm{LuFeCuO}_{4}$. In addition, we found the presence of nano-sized domains, which consist of a short-range ordering of $\mathrm{Fe}^{3+}$ and $\mathrm{Cu}^{2+}$ on the triangular lattice. The present results suggest that the short-range ordering gives rise to some anomalous dielectric properties in $\mathrm{LuFeCuO}_{4}$.
\end{abstract}

Key words: charge ordering, iron oxide, triangular lattice system, transmission electron microscopy

\section{INTRODUCTION}

Ferroelectric materials are used widely as the electric device, because of their ferroelectricity, piezoelectricity, pyroelectricity and electro-optical effect Ferroelectric materials such as $\mathrm{BaTiO}_{3}$ have a spontaneous polarization arising from a coherent arrangement of electric dipoles due to the relative displacement of cations and anions [1,2]. On the other hand, recent works revealed that a ferroelectric polarization in the charge-frustrated system, $\mathrm{LuFe}_{2} \mathrm{O}_{4}$, originates from the charge ordering, which is characterized by a regular arrangement of $\mathrm{Fe}^{2+}$ and $\mathrm{Fe}^{3+}$ ions on the triangular lattice [3].

$\mathrm{LuFe}_{2} \mathrm{O}_{4}$ belongs to the charge-frustrated systems $R \mathrm{Fe}_{2} \mathrm{O}_{4} \quad(R=\mathrm{Ho}-\mathrm{Lu}, \quad \mathrm{Y})$ and has a rhombohedral structure with the space group of $\overrightarrow{\boldsymbol{R}} \boldsymbol{3} \boldsymbol{m}[4]$. In these compounds, triangular lattice planes of irons are stacked along the [001] direction and the Fe-O plane is called W-layer, as shown in Fig.1. Because the average valence of $\mathrm{Fe}$ ions is +2.5 in $\mathrm{LuFe}_{2} \mathrm{O}_{4}, \mathrm{Fe}^{3+}$ and $\mathrm{Fe}^{2+}$ ions coexist on the triangular lattice in the $\mathrm{W}$-layer. This coexisting state of $\mathrm{Fe}^{3+}$ and $\mathrm{Fe}^{2+}$ should lead to the spin/charge frustration on the triangular lattice. Recent electron diffraction and neutron diffraction experiments revealed that the relaxation of the charge frustration results in the formation of a charge ordering (CO) on the triangular lattice below $500 \mathrm{~K}$. Note that the $\mathrm{CO}$ structure is characterized by the regular arrangement of $\mathrm{Fe}^{3+}$ and $\mathrm{Fe}^{2+}$ ions $[5,6]$. In addition, it has been reported that the emergence of the spontaneous polarization $\left(\mathrm{P}_{\mathrm{s}}\right)$ is strongly correlated to the formation of the $\mathrm{CO}$ structure and the magnitude of $\mathrm{P}_{\mathrm{s}}$ increases discontinuously at the ferrimagnetic transition temperature of about $250 \mathrm{~K}$ [1]. This suggests that $\mathrm{LuFe}_{2} \mathrm{O}_{4}$ has some interaction between ferroelectric and magnetic orderings.

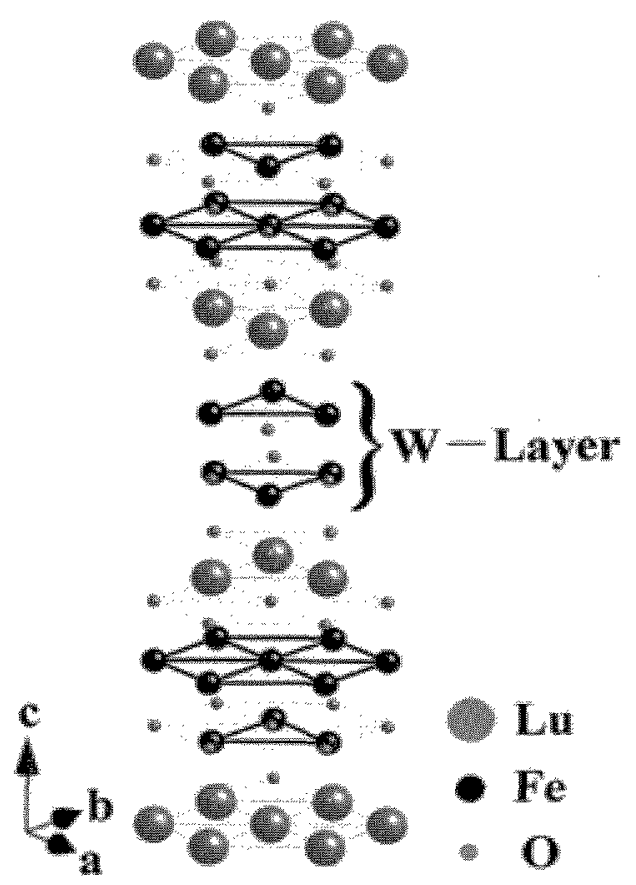

Figure 1, Crystal structure of $\mathrm{LuFe}_{2} \mathrm{O}_{4}$ 
According to a previous work on $\mathrm{RFe} \mathrm{MO}_{4}$ $\left(\mathrm{M} ; \mathrm{Mg}^{2+}, \mathrm{Mn}^{2+}, \mathrm{Co}^{2+}, \mathrm{Cu}^{2+}, \mathrm{Zn}^{2+}\right)$ [7], these compounds show some characteristic magnetic transitions and the transition temperatures are much lower than the ferrimagnetic transition temperature of $\mathrm{LuFe}_{2} \mathrm{O}_{4}$ [8-10]. For instance, $\mathrm{YFeMnO}_{4}$ shows a magnetic transition at around 70K [8]. $\mathrm{LuFeCoO}_{4}$ exhibits a magnetic transition with a cusp at $75 \mathrm{~K}$ due to the anisotropic effects of the $\mathrm{Co}^{2+}$ ion [9]. $\mathrm{LuFeMgO}_{4}$ with the nonmagnetic ion $\left(\mathrm{Mg}^{2+}\right)$ shows a characteristic magnetic fluctuation [11]. Neutron diffraction experiments and Monte Carlo simulation revealed the presence of a local cationic ordering of $\mathrm{Cu}^{2+}$ and $\mathrm{Fe}^{3+}$ in $\mathrm{LuFeMgO}_{4}$ with a short-range coherent length (about 10 atomic length). It was discussed that the magnetic fluctuation in $\mathrm{LuFeMgO}_{4}$ has a close relation with thermal fluctuations and freezing of the short-range magnetically ordered regions (magnetic clusters) [12]. Furthermore, it was reported that $\mathrm{YbFeMnO}_{4}$ has a static magnetic short-range ordered structure with a two-dimensional character, which was characterized by a magnetic superlattice structure with the wave vector $\mathrm{q}=[1 / 3$ $1 / 3 l][13]$. In this work, in order to clarify the short-tange ordered (SRO) structure in $R \mathrm{Fe} M \mathrm{O}_{4}$, we will pay attention to the $\mathrm{LuFeCuO}_{4}$ compound and carefully investigated microstructures related to the magnetic/dielectric properties mainly by means of the transmission electron microscopy (TEM).

\section{EXPERIMENTAL METHOD}

$\mathrm{LuFeCuO}_{4}$ polycrystalline samples were prepared by a conventional solid-state reaction method. Samples for the TEM observation were prepared by $\mathrm{Ar}^{+}$-ion milling method. The TEM observation was carried out with JEM-2010 TEM in a wide temperature window between $100 \mathrm{~K}$ and $700 \mathrm{~K}$. The complex dielectric constant was measured by LCR-meter (hp-4284A) in the temperature range between $293 \mathrm{~K}$ and $600 \mathrm{~K}$.

\section{RESULTS AND DISCUSSION}

In Fig. 2, we displayed typical electron diffraction (ED) patterns of $\mathrm{LuFeCuO}_{4}$ at room temperature. Note that we indexed the diffraction spots in Fig. 2 as a hexagonal unit cell notation. As shown by arrows (A) and (B) in Figs. 2(a) and 2(b), respectively, spotty diffuse scatterings with weak intensity can be seen around the $(1 / 3-\delta 1 / 3-\delta 0)$-type $(\delta=0.06)$ positions. On the other hand, as shown by an arrow (C) in Fig. 2(c), zigzag-shaped diffuse streaks with intensity maxima can be seen around the $\left(\begin{array}{lll}1 / 3 & 1 / 3 & l\end{array}\right)$ positions. Thus, we examined the configuration of these diffuse scatterings in the reciprocal space by obtaining various ED patterns at room temperature and determined the distribution of the diffraction spots and diffuse scatterings in the reciprocal lattice of $\mathrm{LuFeCuO}_{4}$, as shown Fig. 3(a). As clearly evident in Fig. 3(b), there appear spotty diffuse scatterings around the $(1 / 3-\delta 1 / 3-\delta i)$ positions, where the magnitude of the deviation $(\delta)$ from the $(1 / 31 / 3$ 0 )-type positions is estimated to be about 0.06 . On the other hand, there exist diffuse streaks with weak intensity elongating along the approximately [11 12] direction. From a careful analysis of the diffuse scatterings, it can be considered that a SRO structure with the modulation vector $\mathrm{q}=[1 / 3-\delta 1 / 3-\delta 0](\delta=0.06)$ is formed in $\mathrm{LuFeCuO}_{4}$.

Then, in order to clarify what gives rise to these characteristic diffuse scatterings, we tried to obtain high-resolution (HR) lattice images at room temperature. From the analysis of the HR lattice images, it is revealed
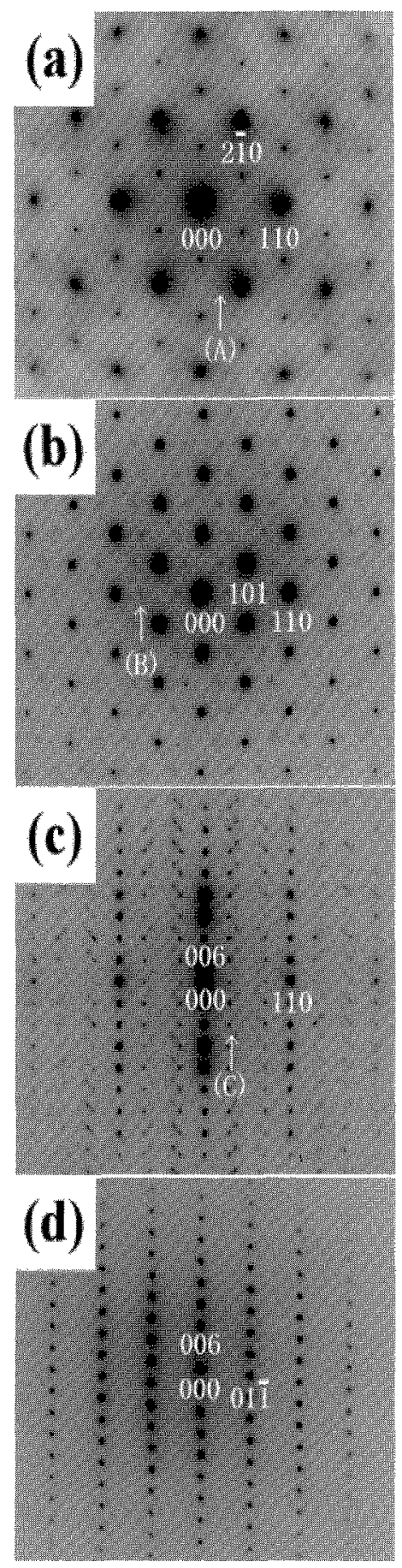

Fig. 2. Electron diffraction patterns of $\mathrm{LuFeCuO}_{4}$ taken along the (a) $[001]$, (b) $[\overline{1} 11]$, (c) $[1 \overline{1} 0]$ and (d) $[\overline{100}]$ zone-axis directions at room temperature, respectively. 
that there exists a characteristic short-range ordering of $\mathrm{Fe}^{3+}$ and $\mathrm{Cu}^{2+}$ ions on the triangular lattice. The details have been reported in Ref. [14]. In addition, in order to elucidate the spatial distribution of the SRO structure in the real space, we obtained a dark-field (DF) image using a spotty diffuse scattering at $(1 / 3-\delta 1 / 3-\delta 0)$ $(\delta=0.06)$ position. As understood in Fig. 4 , a bright dot-shaped contrast with the size of $5-10 \mathrm{~nm}$ can be seen. This suggests that there exist nano-sized domains with the $5-10 \mathrm{~nm}$ size, which originates from the short-range ordering of the $\mathrm{Fe}^{3+}$ and $\mathrm{Cu}^{2+}$ ions. From these results, the substitution of $\mathrm{Cu}^{2+}$ for $\mathrm{Fe}^{2+}$ suppresses the long-ranged $\mathrm{CO}$ structure and forms the nano-sized domains with the $5-10 \mathrm{~nm}$ size. It should be noted that the presence of the nano-scaled domains in $\mathrm{LuFeCuO}_{4}$ is

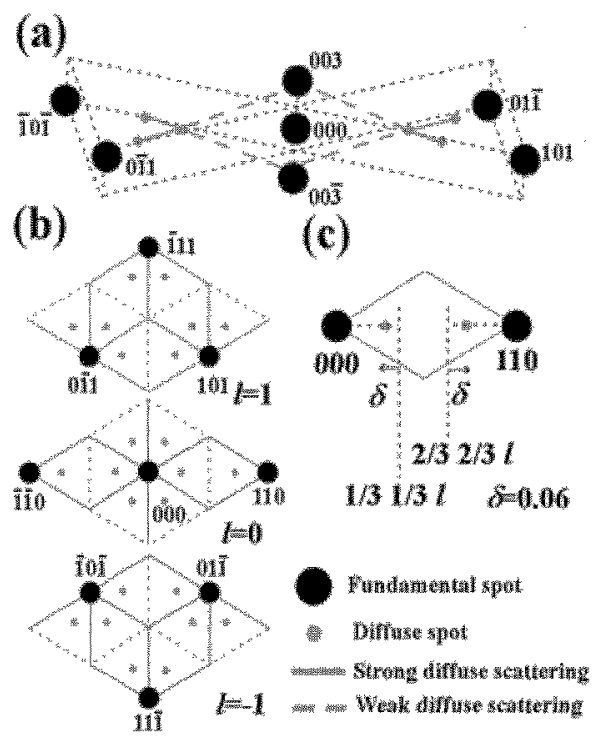

Fig. 3. Schematic representation of the reciprocal lattice. (a) Reciprocal lattice, (b) Cross-section diagram and (c) Arrangement of diffuse spot, respectively.

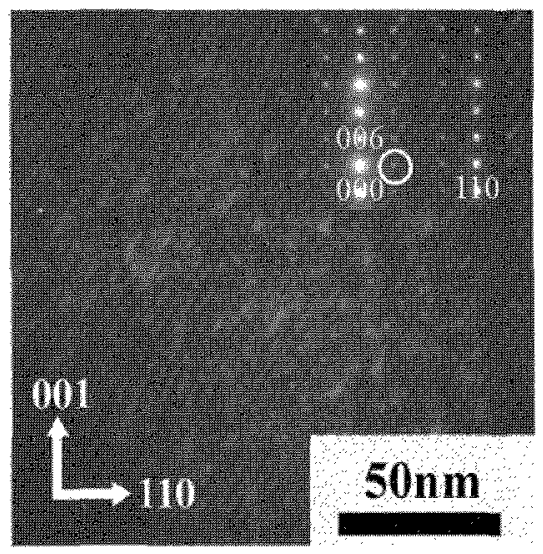

Fig. 4. Dark-field image obtained by using the spotty diffuse scattering indicated by the white circle in the ED pattern at room temperature. (inset) Electron diffraction pattern with the [1] 0$]$ incidence.

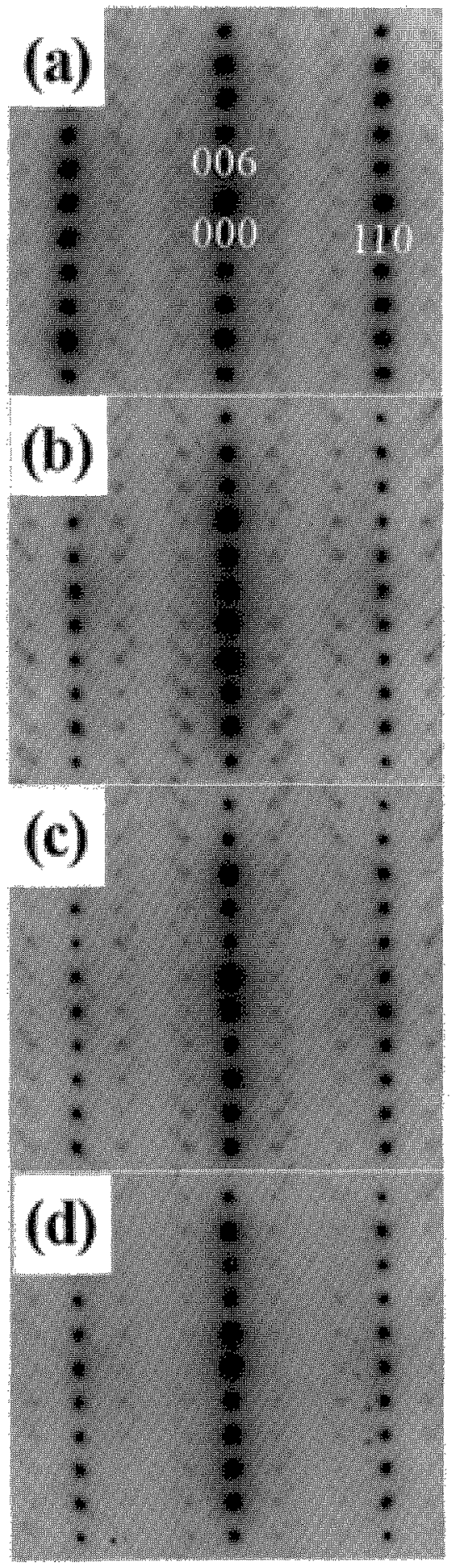

Fig. 5. Temperature dependence of electron diffraction patterns with the $[1 \overline{1} 0]$ incidence obtained at (a) $100 \mathrm{~K}$, (b) $298 \mathrm{~K}$, (c) $408 \mathrm{~K}$ and (d) $641 \mathrm{~K}$, respectively.

similar to that of the magnetic clusters in $\mathrm{LuFeMgO}_{4}$ [12]. In addition, the temperature dependence of the magnetization in $\mathrm{LuFeCuO}_{4}$ exhibits a similar behavior to that in $\mathrm{LuFeMgO}_{4}$ [10]. The nano-sized domains originating from the short-range ordering of $\mathrm{Fe}^{3+}$ and $\mathrm{Cu}^{2+}$ ions on the triangular lattice should be strongly related to the magnetic clusters. On the other hand, it is found that $\mathrm{LuFeCuO}_{4}$ shows a characteristic low- 
frequency dielectric dispersion around $300 \mathrm{~K}$. We analyzed it using the Debye model and found that $\mathrm{LuFeCuO}_{4}$ is a dielectric compound with an orientational polarization [15].This suggests that the ordering of $\mathrm{Fe}^{3+}$ and $\mathrm{Cu}^{2+}$ ions on the triangular lattice gives rise to an electric dipole in each of the nano-sized domains. In addition, in order to elucidate temperature change of the nano-sized domains on warming, we examined the temperature variation of the ED patterns in $\mathrm{LuFeCuO}_{4}$. Figure 5 shows the temperature variation of the ED pattern with the $[1 \overline{1} 0]$ zone axis. Though intensities of both the spotty diffuse scatterings and the diffuse streaks become weak as the temperature is increased, zigzag-shaped diffuse scattering with intensity maxima can be still seen around $641 \mathrm{~K}$. This suggests that the nano-sized domains in $\mathrm{LuFeCuO}_{4}$ are static. The static nano-sized domains in $\mathrm{LuFeCuO}_{4}$ are similar to the static magnetic clusters in $\mathrm{YbFeMnO}_{4}$ [12]. The present results suggest that the nano-sized domains in $\mathrm{LuFeCuO}_{4}$ have a close relation to the characteristic magnetic/dielectric properties. The nano-sized domains are characterized as the static clusters with both the short-range correlation and the orientational polarization.

\section{CONCLUSION}

In summary, we investigated diluting effects on the microstructures with respect to the magnetic/dielectric properties mainly by substituting $\mathrm{Fe}^{2+}$ for $\mathrm{Cu}^{2+}$ ions in $\mathrm{LuFe}_{2} \mathrm{O}_{4}$. The substitution suppresses the formation of a long-ranged $\mathrm{CO}$ structure in $\mathrm{LuFe}_{2} \mathrm{O}_{4}$ and forms static nano-sized domains, which originates from a short-range ordering of $\mathrm{Fe}^{3+}$ and $\mathrm{Cu}^{2+}$ ions. In addition, it is suggested that this short-range ordered structure of $\mathrm{Fe}^{3+}$ and $\mathrm{Cu}^{2+}$ ions causes the electric and magnetic dipoles of the nano-sized domains and gives rise to some anomalous dielectric properties in $\mathrm{LuFeCuO}_{4}$.

\section{Acknowledgement}

This work was supported by Grant-in-Aid for Scientific Research on Priority Areas "Novel States of Matter Induced by Frustration" (19052002) from Ministry of Education, Culture, Sports, Science and Technology. One of the authors (S.M) was supported by the Murata Scientific Foundation.

\section{References}

[1] Kittel, C. Introduction to Solid State Physics (wiley, New York) (1995)

[2] R.E. Cohen and H.Krakauer, Phys.Rev.B 42, 6416-6423 (1990)

[3]N.Ikeda, H.Ohsumi, K.Owada, K.Ishii, T.Inami, Y.Murakami, K.Kakurai, K.Yoshii, S.Mori, Y.Horibe and H.Kito, Nature(London), 436, 1136-1138 (2005)

[4]N.Kimizuka, A.Takenaka, Y.Sasada and T.Katsura, Solid State Commun. 15, 1321-1323 (1975)

[5] Y.Yamada, S.Nohdo and N.Ikeda, J. Phys. Soc. Jpn. 66, 3733-3736 (1997)

[6] Y.Yamada, K.Kitsuda,S.Nohdo and N.Ikeda, Phys.Rev.B 62,12167-12174 (2000)
[7] N.Kimizuka and E.Takayama, J. Solid State Chem. 40, 109-116 (1981)

[8] J.Iida, M.Tanaka, and Y.Nakagawa, J. Phys. Soc. Jpn. 59, 4443-4448 (1990)

[9] T.Sunaga, M.Tanaka, N.Sakai and Y.Tsunoda, J. Phys. Soc. Jpn. 70, 3713-3718 (2001)

[10] K.Yoshii, N.Ikeda, Y.Matsuo, Y.Horibe and S.Mori, Phys.Rev.B 76 (2), 024423 (2007)

[11] N.Ikeda,K.Kohn,E.Himoto and M. Tanaka, J. Phys. Soc. Jpn. 64, 4371-4377 (1995)

[12] Y.Todate, E.Himoto, C.Kikuta, M.Tanaka and J.Suzuki, Phys.Rev.B 57,485-491 (2000)

[13]J.Tida, M.Tanaka, S.Funahashi and Y.Nakagawa, J.Appl.Phys. 69(8) 5801-5803

[14]Y.Matsuo, Y.Horibe, S.Mori, K.Yoshii and N.Ikeda, J.Mag.Mag.Mater. 310, e349-e351 (2007)

[15] Y.Matsuo, K.Yoshii, N.Ikeda and S.Mori (unpublished).

(Received December 8, 2007; Accepted January 31, 2008) 